\title{
Complete the Following Statement:
}

\section{Industry-Sponsored Antiepileptic Drug Pregnancy Registries Provide Information that is Beneficial to:}

\author{
Patients \\ Doctors \\ The Sponsor \\ All of the Above \\ None of the Above \\ Cannot Respond Due to Risk of COI
}

Final Results From 18 Years of the International Lamotrigine Pregnancy Registry.

Cunnington MC, Weil JG, Messenheimer JA, Ferber S, Yerby M, Tennis P. Neurology 2011;76:1817-1823.

OBJECTIVE: To monitor for a signal for major teratogenicity following in utero lamotrigine exposure. METHODS: Health care providers reported lamotrigine exposure during pregnancy, and subsequent outcomes, on a voluntary basis. Prospective reporting early in pregnancy was encouraged. Major congenital malformations (MCMs) were classified according to the Centers for Disease Control and Prevention (CDC) criteria and were reviewed by a pediatrician on the Registry's Scientific Advisory Committee. The proportion of infants with MCMs was calculated by trimester and therapy type and descriptively compared to population-based reference estimates. RESULTS: Over an 18-year period, 35 infants with MCMs were observed among 1,558 first-trimester monotherapy exposures: 2.2 percent (95\% confidence interval [CI] 1.6\%-3.1\%). This was similar to estimates from general population-based cohorts. The observed proportion of infants with MCMs among 150 lamotrigine/valproate polytherapy exposures was 10.7 percent (95\% Cl 6.4\%-17.0\%) and was 2.8 percent (95\% Cl 1.5\%-5.0\%) among 430 infants exposed to lamotrigine polytherapy without valproate. No consistent pattern of malformation type, or malformation frequency by dose, was observed. DISCUSSION: The Registry did not detect an appreciable increase in MCM frequency following firsttrimester lamotrigine monotherapy exposure. With over 1,500 first-trimester monotherapy exposures, the Registry was powered to detect major teratogenicity. The proportion of infants with MCMs following lamotrigine/valproate polytherapy exposure was high, but similar to that previously reported with valproate monotherapy. The Registry failed to observe an increased MCM frequency with increasing lamotrigine dose. Monitoring of specific malformations among lamotrigine-exposed pregnancies will continue through case-control surveillance in the European Congenital Anomalies and Twins Registers network.

Epilepsy Currents, Vol. 11, No. 6 (November/December) 2011 pp. 181-183

○ American Epilepsy Society

OPEN 2 ACCESS Freely available online

\section{Commentary}

GlaxoSmithKline (GSK) put forth their final results of the International Lamotrigine Pregnancy Registry, reporting major congenital malformations (MCMs) in 35 infants out of 1,558 first trimester monotherapy exposures, a frequency of $2.2 \%$ 
(95\% confidence interval [CI] of 1.6-3.1\%) (1). This international registry has been enrolling since 1992 , with $65 \%$ of subjects enrolled from the United States (Lamictal was approved in the United States in late 1994), and 20\% more from northern Europe. The enrollment was closed in June 2009, since the proscribed enrollment goal of 1,000 first-trimester lamotrigine exposures was met. This enrollment target was thought to provide adequate power to identify a large increase in the frequency over the expected rate of all combined MCMs with lamotrigine exposure.

In the early 1990s, Glaxo Wellcome launched five international pregnancy registries: 1) the Acyclovir Pregnancy Registry, 2) the Antiretroviral Pregnancy Registry, 3) the Lamotrigine Pregnancy Registry, 4) the Sumatriptan Pregnancy Registry, and 5) the Bupropion Pregnancy Registry, with the aim of obtaining information about rates of teratogenicity of these compounds (2). The International Lamotrigine Pregnancy Registry has been lauded by the epilepsy community (although it enrolled subjects taking lamotrigine for reasons other than epilepsy); it was started early in the marketed use of lamotrigine, and therefore was definitely a departure from the postmarketing activities for other newer antiepileptic drugs (AEDs) for which teratogenic risk in humans was an afterthought or completely ignored. In 2002, with 9 years of data, GSK published their preliminary results (3). (An even more preliminary report was published in 2000 of the results of all five registries above [4]). They reported the occurrence of three infants with MCMs (1.8\%) out of 168 first-trimester lamotrigine exposure $(95 \% \mathrm{Cl}$ $0.5-5.5 \%)(3)$.

In 2005, in another update, GSK reported that of 414 first-trimester exposures to lamotrigine monotherapy, 12 outcomes with MCMs occurred (2.9\%, 95\% Cl 1.6-5.1\%) (5). Another analysis was published in 2007 , in which the frequency of MCMs in 802 first-trimester monotherapy lamotrigine exposures was $2.7 \%$ (95\% Cl 1.8-4.2\%) (6). The main point of this particular manuscript, however, was to document the absence of a dose-dependent relationship with MCMs, which was analyzed by logistic regression using 100-mg increments, up to $400 \mathrm{mg}$ per day. This GSK publication appeared to be in response to a report from the U.K. Pregnancy Register in 2006 (7), in which a lamotrigine dose-dependent relationship with MCMs between the use of less versus more than $200 \mathrm{mg}$ per day was found. An increased risk of isolated facial clefts in exposed infants was pointedly not found in the final GSK registry report, refuting what was first reported by the North American AED Pregnancy Registry (8) but later not borne out in a case control study from the EUROCAT database (9).

The frequency of malformations in the GSK registry has basically been low at all assessment points during the registry, from 1.8 to 2.9 percent, with the final number at $2.2 \%$ surrounded by very narrow confidence intervals, which have also gradually been closing in around this number over the years, now at 1.6 to 3.1 percent. However, this confidence interval means that it is just as likely that the "true" frequency of malformations can be $3.1 \%$ or $1.6 \%$ as it is $2.2 \%$. The authors compare this result to MCM frequencies in the general population from large surveillance databases, of 1.6 to 2.2 percent. The GSK results are similar to findings from the EURAP registry, in which the frequency of MCMs with first-trimester lamotrigine monotherapy exposure was $2.3 \%$, with 30 infants of 1,280 exposures having MCMs at 2 months follow-up; seven additional MCMs were found in this cohort after 1 year of follow-up (10). Therefore, the frequency of malformations in the GSK registry is consistent over 18 years, very close to the general population and nearly identical to that of a concurrently reported academic database. The internal consistency and favorable comparisons to external databases support the validity of the GSK registry findings.

Of interest, another very recent report from a Danish population-based database found a frequency of MCMs of $3.7 \%$ in infants exposed to lamotrigine in the first trimester (38 of 1,019 exposures) (11). After the authors adjusted for the diagnosis of epilepsy, the risk of MCMs for all AED exposures was decreased; overall, the presence of an epilepsy diagnosis itself contributed about a 40 to 50 percent increased frequency of birth defects compared with the non-AED-exposed comparator group. Further, after adjusting for an epilepsy diagnosis, the risk of MCMs with lamotrigine exposure compared with controls was not significant. This may be the best data thus far to quantitate a risk imparted by epilepsy itself.

Limitations of the GSK study methodology include the risk of reporting bias, since women were referred to the registry by a health care provider and enrolled voluntarily, whether they knew any ultrasound results or not at the time of enrollment. It is unclear if this enrollment methodology would bias the study toward a lower MCMs frequency. The results would suggest not; there were three cases of anencephaly reported, and this malformation is reliably seen on ultrasound between 10 and 14 weeks of pregnancy. This surprising finding and its association with lamotrigine should be further assessed using the EUROCAT data as well. The GSK registry enrollment strategy is similar to that of the EURAP registry; neither registry is population representative and may even include some common subjects. The low frequency of MCMs in the GSK final report is consistent with the stated methodology of including birth defects found only before hospital discharge. This frequency of malformations is identical to that found in the North American Pregnancy Registry strategy, which also uses a very short period after birth to assess MCMs, but is shorter than the EURAP assessments, which are at 2 months and up to 1 year. Although there was no concurrent control group in the GSK study, the data within the cohort are revealing and consistent; a higher frequency of birth defects with polytherapy that includes valproate has been consistently reported, now at $10.7 \%$ in this final report.

The issue of a lamotrigine dose-dependent association with MCM frequency is not sorted out. The GSK registry did not detect it; however, the EURAP report showed an increase in MCMs with doses $\geq 300 \mathrm{mg}$ per day. It is intuitive that a greater exposure would pose a greater risk, and this is certainly present for valproate exposure. This relationship is more clearly detected with valproate because the frequency of adverse events is in general much greater.

GSK has positioned lamotrigine as an AED to consider when treating women of childbearing potential. In spite of the challenges around the variable clearance of lamotrigine with exogenous hormones such as oral contraceptives and with endogenous hormones during pregnancy, the GSK pregnancy 
registry and other registries bear out a low frequency of MCMs with use during pregnancy. This is good and useful news for patients, healthcare providers, and for GSK. Did we need an industry-sponsored pregnancy registry to find this out? These findings would have eventually been reported in other registries. However, putting aside the marketing strategy, this registry was established before any of the academic registries, which, outside of the national health registries are supported by industry funding in any case. Even the Danish database study cited herein was supported in part by Lundbeck. The GSK registry was established to answer a dire clinical question, provided early reports on frequencies of malformations, was not more biased than other registries, and of course helped to market Lamictal. Best answer D.

\section{by Cynthia L. Harden, MD}

\section{References}

1. Cunnington MC, Weil JG, Messenheimer JA, Ferber S, Yerby M, Tennis P. Final results from 18 years of the International Lamotrigine Pregnancy Registry. Neurology 2011;76:1817-1823.

2. White AD, Andrews EB. The Pregnancy Registry program at Glaxo Wellcome Company. J Allergy Clin Immunol 1999;103:S362-363.

3. Tennis P, Eldridge RR; International Lamotrigine Pregnancy Registry Scientific Advisory Committee. Preliminary results on pregnancy outcomes in women using lamotrigine. Epilepsia 2002;43:11611167.

4. Reiff-Eldridge R, Heffner CR, Ephross SA, Tennis PS, White AD, Andrews EB. Monitoring pregnancy outcomes after prenatal drug exposure through prospective pregnancy registries: A pharmaceutical company commitment. Am J Obstet Gynecol 2000;182:159-163.

5. Cunnington $M$, Tennis $P$; International Lamotrigine Pregnancy Registry Scientific Advisory Committee. Lamotrigine and the risk of malformations in pregnancy. Neurology 2005;64:955-960.

6. Cunnington M, Ferber S, Quartey G; International Lamotrigine Pregnancy Registry Scientific Advisory Committee. Effect of dose on the frequency of major birth defects following fetal exposure to lamotrigine monotherapy in an international observational study. Epilepsia 2007;48:1207-1210.

7. Morrow J, Russell A, Guthrie E, Parsons L, Robertson I, Waddell R, Irwin B, McGivern RC, Morrison PJ, Craig J. Malformation risks of antiepileptic drugs in pregnancy: A prospective study from the UK Epilepsy and Pregnancy Register. J Neurol Neurosurg Psychiatry 2006;77:193-198.

8. Holmes LB, Baldwin EJ, Smith CR, Habecker E, Glassman L, Wong SL, Wyszynski DF. Increased frequency of isolated cleft palate in infants exposed to lamotrigine during pregnancy. Neurology 2008;70:21522158.

9. Dolk H, Jentink J, Loane M, Morris J, de Jong-van den Berg LT; EUROCAT Antiepileptic Drug Working Group. Does lamotrigine use in pregnancy increase orofacial cleft risk relative to other malformations? Neurology 2008;71:714-722.

10. Tomson T, Battino D, Bonizzoni E, Craig J, Lindhout D, Sabers A, Perucca E, Vajda F; for the EURAP study group. Dose-dependent risk of malformations with antiepileptic drugs: An analysis of data from the EURAP epilepsy and pregnancy registry. Lancet Neurol 2011;10:609-617.

11. Molgaard-Nielsen D, Hviid A. Newer-generation antiepileptic drugs and the risk of major birth defects. JAMA 2011;305:1996-2002. 